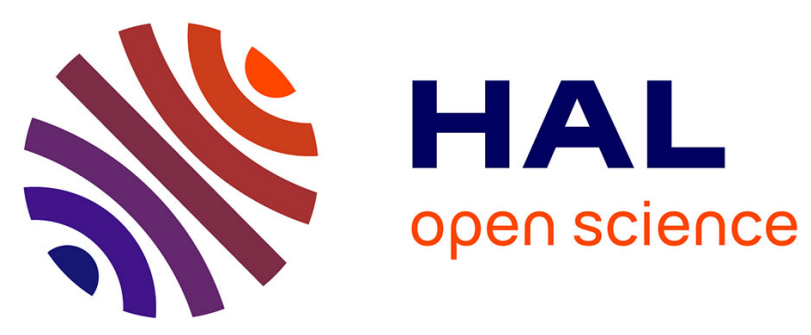

\title{
PREVIEWED IMPEDANCE ADAPTATION TO COORDINATE UPPER-LIMB TRAJECTORY TRACKING AND POSTURAL BALANCE IN DISTURBED CONDITIONS
}

\author{
Aurélien Ibanez, Philippe Bidaud, Vincent Padois
}

\section{To cite this version:}

Aurélien Ibanez, Philippe Bidaud, Vincent Padois. PREVIEWED IMPEDANCE ADAPTATION TO COORDINATE UPPER-LIMB TRAJECTORY TRACKING AND POSTURAL BALANCE IN DISTURBED CONDITIONS. Kenneth J Waldron, Mohammad O Tokhi, Gurvinder S Virk. NatureInspired Mobile Robotics - Proceedings of the 16th International Conference on Climbing and Walking Robots and the Support Technologies for Mobile Machines, World Scientific, pp.519-528, 2013, 10.1142/9789814525534_0066. hal-01031868

\section{HAL Id: hal-01031868 \\ https://hal.science/hal-01031868}

Submitted on 22 Jul 2014

HAL is a multi-disciplinary open access archive for the deposit and dissemination of scientific research documents, whether they are published or not. The documents may come from teaching and research institutions in France or abroad, or from public or private research centers.
L'archive ouverte pluridisciplinaire HAL, est destinée au dépôt et à la diffusion de documents scientifiques de niveau recherche, publiés ou non, émanant des établissements d'enseignement et de recherche français ou étrangers, des laboratoires publics ou privés. 


\title{
Previewed impedance adaptation to coordinate upper-limb trajectory tracking and postural balance in disturbed conditions
}

\author{
A. IBANEZ*, P. BIDAUD and V. PADOIS \\ ISIR Institute for Intelligent Systems and Robotics UMR222, \\ CNRS-UPMC Université Pierre et Marie Curie \\ Paris, 75005, France \\ *E-mail: $\{$ ibanez, bidaud, padois\}@isir.upmc.fr
}

\begin{abstract}
This paper proposes a preview control method for the whole-body motion of humanoid robots ensuring high performance in both interaction and postural balance tasks under large physical perturbations. By previewing the reduced coupled models of upper-limb interaction and postural balance dynamics, the proposed controller adapts simultaneously the impedance of the arms and the center of mass trajectory with respect to known external perturbations. Here, we show how the ZMP preview control formulation can be extended to account for disturbances resulting from the interacting arms dynamics of which control parameters are adapted online in order to maximize both interaction and balance performances over a preview horizon. The validity of this formulation is assessed through simulation considering a force applied at the humanoid hand level when it is walking.
\end{abstract}

Keywords: Humanoid, Biped walking, Legged robot, Adaptive impedance, Preview control, Zero-Moment Point, Postural balance, Manipulation, Interaction.

\section{Introduction}

Controlling the behavior of a humanoid robot placed in a dynamic environment in which it must perform manipulation tasks requires to ensure a robust control of postural balance and adaptation of handling tasks to make them compatible with the balance constraints.

In the case of human beings the coordination of motor activity can be interpreted ${ }^{1}$ as a dialog in between two different parts of the central nervous system (CNS) involved in motor control: a predictive part relating high-level voluntary actions to carry out reaching a particular goal in a particular environment (which presupposes knowledge about expected action effects) and a reactive part performing local motor parametric ad- 
justments imposed by the needed reactivity to the environment requests and the basic biological needs. The CNS generates Anticipatory Postural Adjustments (APA) that minimize the effects on balance of a simultaneous focal activity, while task-related motor activity adapts to constraints raised by postural balance. The whole motor response is organized with the aim to coordinate the APAs and the focal activity ${ }^{2}$ in order to maximize the overall performance.

To obtain a similar control policy for humanoids, we propose a control scheme based on a multi-objective predictive/preview control of the impedance of the upper limbs and of the postural system.

Among existing contributions in this domain, control methods such as impedance controllers ${ }^{3}$ allows to define the reactive behavior of a manipulator during interaction tasks of which parameters can be defined in an optimal way. ${ }^{4}$ This resulting behavior of the upper-limbs induces perturbations ${ }^{5}$ on the lower-bodies of the robot. The control of these two systems having coupled dynamics and independent objectives can be handled in an anticipative way using a distributed model predictive control (DMPC) method $^{6}$ dynamically tuning reactive control parameters.

The control method proposed in this paper relies on coupled reduced representations of two tasks dynamics, biped balance and upper-limb manipulation, expressed at the center of mass (CoM) and hand levels, respectively. For a given horizon of control parameters, the dynamics of the upper limbs can be previewed under an external force applied at the hand and an induced horizon of disturbance on the supporting platform is estimated, allowing for the evaluation over a finite horizon of a manipulation performance index and a ZMP-based balance stability criterion.

The novelty of the approach proposed in this work and the obtained results are presented in the next sections.

\section{Formulation}

The horizon of control parameters is defined as the horizon of CoM horizontal jerks $\mathbf{u}$ and cartesian control stiffness $K$ and damping $C$ at the hand level. At each control step, an optimization process is run over the control parameters to maximize the performance index of both balance and manipulation tasks over a finite horizon. 


\subsection{Lower-limbs dynamics}

The lower-limbs motor activity mainly supports postural balance. Under this assumption and neglecting vertical and rotational effects we reduce the system to a punctual mass centered at the CoM of the robot of which

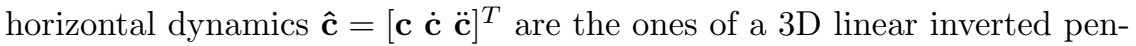
dulum (LIP3D) as seen in the works of Kajita et al. ${ }^{7}$ An horizon of external force $\mathbf{F}^{e, c}$ applied on the CoM is supposed to be known. By rewriting the Zero-Moment Point (ZMP) equations for the LIP3D under $\mathbf{F}^{e, c}$ we can compute its position $\mathbf{p}$ in the ground plane as a function of $\hat{\mathbf{c}}$ and $\mathbf{F}^{e, c}$ : under these hypotheses, the LIP3D model is subject to inertial effects, gravity and external actions, and the ZMP position $\mathbf{p}$ writes

$$
\mathbf{p}=\mathbf{c}-\frac{M z}{M g-\mathbf{F}^{e, c} \mid z} \ddot{\mathbf{c}}+\frac{z}{M g-\mathbf{F}^{e, c} \mid z} \mathbf{F}^{e, c},
$$

where $z$ is the center of mass altitude, supposed in this model to be constant over time, $\mathbf{F}^{e, c}{ }_{\mid z}$ is the vertical coordinate of the force applied on the CoM and $M, g$ are the robot mass and gravity amplitude, respectively.

Balance is assumed to be maintained as long as the ZMP position is inside the convex hull of the contact points on the ground. The maximization of balance stability can be abridged as the minimization of the distance between the ZMP position $\mathbf{p}$ and a reference $\overline{\mathbf{p}}$ which is the center of the sustentation convex hull.

The CoM is controlled, similarly to the works of Kajita et al., ${ }^{7}$ through the computation of desired horizontal input jerk $\mathbf{u}$. Time is discretized over time intervals of constant lengths $\delta t$, and for a continuous function $g$ of time $g_{k}$ denotes $g(k \delta t)$. Given an horizon of $\mathbf{u}_{k}$ the approximated CoM dynamics can be previewed in the following way

$$
\hat{\mathbf{c}}_{k} \equiv\left\{\begin{array}{l}
\mathbf{c}_{k}=\mathbf{c}_{k-1}+\delta t \dot{\mathbf{c}}_{k-1}+\frac{\delta t^{2}}{2} \ddot{\mathbf{c}}_{k-1}+\frac{\delta t^{3}}{6} \mathbf{u}_{k-1} \\
\dot{\mathbf{c}}_{k}=\dot{\mathbf{c}}_{k-1}+\delta t \ddot{\mathbf{c}}_{k-1}+\frac{\delta t^{2}}{2} \mathbf{u}_{k-1} \\
\ddot{\mathbf{c}}_{k}=\ddot{\mathbf{c}}_{k-1}+\delta t \mathbf{u}_{k-1}
\end{array}\right.
$$

A matrix-form of the preview of the ZMP position can be written, as seen in the works of Wieber, ${ }^{8}$ using Eq. (1) and Eq. (2) expressed from current step $k$ to final preview step $k+N$. Let us denote the horizons of previewed ZMP positions $\mathcal{P}_{k} \equiv\left[\begin{array}{lll}\mathbf{p}_{k+1} & \ldots & \mathbf{p}_{k+N}\end{array}\right]^{T}$ and input jerks $\chi_{k}^{\mathbf{c}} \equiv\left[\begin{array}{lll}\mathbf{u}_{k+1} & \ldots & \mathbf{u}_{k+N}\end{array}\right]^{T}$, which brings

$$
\mathcal{P}_{k}=\mathbf{P}_{\mathbf{x}}^{\text {dis }} \hat{\mathbf{c}}_{k}+\mathbf{P}_{\mathbf{u}}^{\text {dis }} \chi_{k}^{\mathbf{c}}+\mathbf{D}_{k}
$$


where, writing $\alpha^{d i s} \equiv \frac{1}{M g-\mathbf{F}^{e, c} \mid z}$,

$\mathbf{P}_{\mathbf{x}}^{\mathrm{dis}} \equiv\left[\begin{array}{ccc}1 & \delta t & \frac{\delta t^{2}}{2}-M z \alpha_{k+1}^{d i s} \\ \vdots & \vdots & \vdots \\ 1 & N d t & N^{2} \frac{\delta t^{2}}{2}-M z \alpha_{k+N}^{d i s}\end{array}\right], \mathbf{P}_{\mathbf{u}}^{\mathbf{d i s}} \equiv\left[\begin{array}{cc}\frac{\delta t^{3}}{6}-\delta t M z \alpha_{k+1}^{d i s} & \cdots 0 \\ \vdots & \ddots 0 \\ \left(1+3 N^{2}\right) \frac{\delta t^{3}}{6}-\delta t M z \alpha_{k+N}^{d i s} & \cdots * *\end{array}\right]$ and finally

$$
\mathbf{D}_{k} \equiv\left[z \mathbf{F}_{k+1}^{e, c} \alpha_{k+1}^{d i s} \cdots z \mathbf{F}_{k+N}^{e, c} \alpha_{k+N}^{d i s}\right]^{T},
$$

which can be interpreted as an horizon of disturbances on the stable ZMP reference $\overline{\mathbf{p}}$.

\subsection{Upper-limbs dynamics}

Upper-limbs dynamics are subject to control parameters and actions from the environment. Manipulation tasks requiring a certain level of precision (with respect to the interaction magnitude involved) are often achieved through controllers of high apparent stiffness, resulting in the transmission of actions through the upper-limbs towards the lower part of the system.

In the case of manipulation, we consider the external actions to be applied at the end-effector, ie the hand of the robot. A PD corrective controller is defined at this point. By comparing the operational space ${ }^{9}$ dynamics of the effector under an external action $\mathbf{F}$ to the free ones, we identify a local impedant model of the hand dynamics ê.

The whole-body control framework used in this work is a LQP-based ${ }^{10}$ controller which computes optimal joint torques with respect to desired joint and cartesian accelerations and constraints, using joint-space dynamics subject to contact wrenches.

To capture the behavior of the upper-limbs, we reduce the humanoid system to a fixed-base manipulator (free-floating joints and contact forces are not considered). In the case of manipulation, given a desired acceleration $\mathbf{a}$

$$
\mathbf{a}=K\left(\mathbf{e}^{*}-\mathbf{e}\right)+C\left(\dot{\mathbf{e}}^{*}-\dot{\mathbf{e}}\right)+\ddot{\mathbf{e}}^{*}
$$

where $K$ and $C$ denote respectively cartesian control stiffness and damping, and $\overline{\mathbf{e}}=\left[\mathbf{e}^{*} \dot{\mathbf{e}}^{*} \ddot{\mathbf{e}}^{*}\right]$ are the reference dynamics of the effector, whole-body control consists in finding joint torques $\tau$ that satisfy the free dynamics

$$
\mathbf{H}(\mathbf{q}) \ddot{\mathbf{q}}^{\mathbf{a}}+\mathbf{N}(\mathbf{q}, \dot{\mathbf{q}})+\mathbf{G}(\mathbf{q})=\boldsymbol{\tau},
$$

where $\mathbf{H}, \quad \mathbf{N}$ and $\mathbf{G}$ denote the joint-space mass, coriolis/centrifugal and gravity matrices, respectively. Dependency to $(\mathbf{q}, \dot{\mathbf{q}})$ is dropped in the rest of 
the paper by linearization of Eq. (5) around current joint state. Joint-space dynamics under $\mathbf{F}$, however, write with $\mathbf{J}_{e}$ the Jacobian of the hand

$$
\mathbf{H} \ddot{\mathbf{q}}+\mathbf{N}+\mathbf{G}=\boldsymbol{\tau}+\mathbf{J}_{e}^{T} \mathbf{F} .
$$

Comparing Eq. (6) to Eq. (5) in the operational space under input joint torques $\boldsymbol{\tau}$ captures the local dynamics $\ddot{\mathbf{e}}$ of the effector subject to action $\mathbf{F}$

$$
\mathbf{M}_{e} \ddot{\mathbf{e}}=\mathbf{F}-\mathbf{M}_{e} \dot{\mathbf{J}}_{e} \mathbf{J}_{e}^{\dagger}(\dot{\mathbf{e}}-\boldsymbol{\nu})+\mathbf{K}_{e}\left(\mathbf{e}^{*}-\mathbf{e}\right)+\mathbf{C}_{e}\left(\dot{\mathbf{e}}^{*}-\dot{\mathbf{e}}\right)+\mathbf{M}_{e} \ddot{\mathbf{e}}^{*},
$$

where $\mathbf{M}_{e}=\left(\mathbf{J}_{e} \mathbf{H}^{-1} \mathbf{J}_{e}^{T}\right)^{-1}, \mathbf{K}_{e}=K \mathbf{M}_{e}, \mathbf{C}_{e}=C \mathbf{M}_{e}$ and

$$
\boldsymbol{\nu} \quad \text { s.t. } \quad \boldsymbol{\nu}=\mathbf{J}_{e} \dot{\mathbf{q}}^{\mathbf{a}} \quad \text { and } \dot{\mathbf{q}}^{\mathbf{a}} \quad \text { s.t. } \quad \mathbf{a}=\mathbf{J}_{e} \ddot{\mathbf{q}}^{\mathbf{a}}+\dot{\mathbf{J}}_{e} \dot{\mathbf{q}}^{\mathbf{a}} \text {. }
$$

The model described in Eq. (7) exhibits terms related to inertial effects, external actions and impedant behavior. We assume the latter to be supported by the lower-limbs, considering that upper and lower models of the robot are serialized. That is,

$$
\mathbf{F}^{e, c}=\mathbf{M}_{e} \dot{\mathbf{J}}_{e} \mathbf{J}_{e}^{\dagger}(\dot{\mathbf{e}}-\boldsymbol{\nu})-\mathbf{K}_{e}\left(\mathbf{e}^{*}-\mathbf{e}\right)-\mathbf{C}_{e}\left(\dot{\mathbf{e}}^{*}-\dot{\mathbf{e}}\right)-\mathbf{M}_{e} \ddot{\mathbf{e}}^{*},
$$

where $\mathbf{J}_{e}^{\dagger}$ is the generalized inverse of the effector jacobian, written here as the dynamically consistent pseudoinverse minimizing kinetic energy. ${ }^{9}$

The following integration scheme is used

$$
\left\{\begin{array}{l}
\mathbf{e}_{k}=\mathbf{e}_{k-1}+\delta t \dot{\mathbf{e}}_{k-1}+\frac{\delta t^{2}}{2} \ddot{\mathbf{e}}_{k-1} \\
\dot{\mathbf{e}}_{k}=\dot{\mathbf{e}}_{k-1}+\delta t \ddot{\mathbf{e}}_{k-1} \\
\ddot{\mathbf{e}}_{k}=\mathbf{M}_{e}^{-1} \mathbf{F}_{k}+\mathbf{a}_{k}-\dot{\mathbf{J}}_{e} \mathbf{J}_{e}^{\dagger}\left(\dot{\mathbf{e}}_{k}-\boldsymbol{\nu}_{k}\right) \text { from Eq. (7), }
\end{array}\right.
$$

where $\boldsymbol{\nu}_{k}$ is derived from Eq. (7) expressed at step $k-1$

$$
\boldsymbol{\nu}_{k}=\dot{\mathbf{e}}_{k-1}+\left(\mathbf{M}_{e} \dot{\mathbf{J}}_{e} \mathbf{J}_{e}^{\dagger}\right)^{-1}\left[\mathbf{M}_{e}\left(\ddot{\mathbf{e}}_{k-1}-\mathbf{a}_{k-1}\right)-\mathbf{F}_{k-1}\right] .
$$

Effector acceleration can hence be written, for $k>0$

$$
\ddot{\mathbf{e}}_{k}=\ddot{\mathbf{e}}_{0}+\mathbf{M}_{e}^{-1}\left(\mathbf{F}_{k}-\mathbf{F}_{0}\right)+\left(\mathbf{a}_{k}-\mathbf{a}_{0}\right)-\dot{\mathbf{J}}_{e} \mathbf{J}_{e}^{\dagger}\left(\dot{\mathbf{e}}_{k}-\dot{\mathbf{e}}_{0}\right)
$$

which allows to preview the effector dynamics over a finite horizon, knowing a future estimation of $\mathbf{F}_{k}$ applied on the hand.

\section{Whole system preview control}

The previewed performance of both balance and manipulation tasks under an external force $\mathbf{F}$ is optimized over a finite horizon. By serializing both upper and lower-limbs reduced models, their dynamics are coupled through the transmission of the external action, relatively to the control stiffness and damping $K, C$ of the effector. The behavior of the resulting coupled model 
is previewed over a horizon of $N$ time steps. Optimal control parameters are deduced from the preview and are set as active control parameters of both balance and manipulation reactive tasks, as shown in Fig. 1.

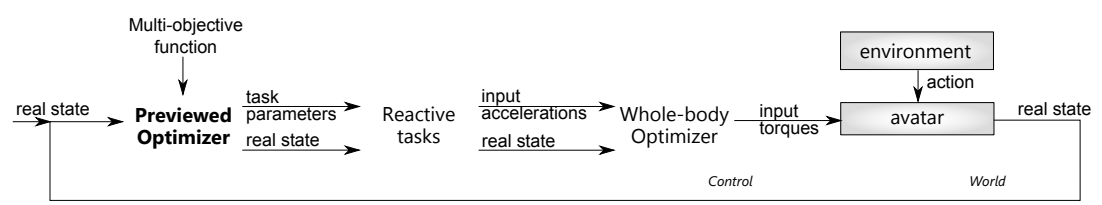

Fig. 1. Control architecture

\subsection{Optimization problem}

At each control step $k$, the manipulation and balance errors $\delta^{\mathbf{e}}$ and $\delta^{\mathbf{p}}$ are minimized for each preview step $i \in[k+1, k+N]$. If $\overline{\mathbf{e}}_{i}$ and $\overline{\mathbf{p}}_{i}$ denote respectively the objective of the manipulation and balance tasks, the following errors $\delta_{i}^{\mathbf{e}} \equiv\left\|\hat{\mathbf{e}}_{i}-\overline{\mathbf{e}}_{i}\right\|^{2}$ and $\delta_{i}^{\mathbf{p}} \equiv\left\|\mathbf{p}_{i}-\overline{\mathbf{p}}_{i}\right\|^{2}$ are to be minimized. To define the relative priority between these two objectives and capture the difference in magnitude of the two errors, a cost function of the form $\omega_{\mathbf{e}} \delta_{i}^{\mathbf{e}}+\omega_{\mathbf{p}} \delta_{i}^{\mathbf{p}}$ is minimized at each control step $k$, for each preview step $i \in[k+1, k+N]$. To enforce the stability of the minimization process we define a quadratic regularization term $h$ and build the following cost function

$$
f_{k}\left(\chi_{k}\right)=\sum_{i=1}^{N}\left(\omega_{\mathbf{e}} \delta_{k+i}^{\mathbf{e}}+\omega_{\mathbf{p}} \delta_{k+i}^{\mathbf{p}}+h_{k+i}\right)
$$

which is minimized with respect to control parameters $\chi_{k} \in \mathbb{R}^{4 N \times 1}$

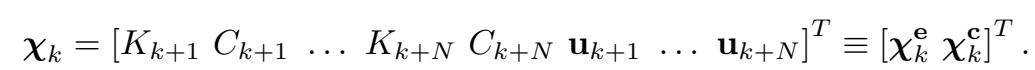

Regularization is achieved by minimizing the CoM input amplitude and the euclidean distance of manipulation control parameters $K, C$ to a reference $\bar{K}, \bar{C}$, that is $h_{i}=\omega_{\mathbf{e}}^{h}\left(\left\|K_{i}-\bar{K}_{i}\right\|^{2}+\left\|C_{i}-\bar{C}_{i}\right\|^{2}\right)+\omega_{\mathbf{p}}^{h}\left\|\mathbf{u}_{i}\right\|^{2}$.

\subsection{Resolution}

To reduce the dimensionality of the problem from $4 N$ to $2 N$, optimal input jerks $\chi_{k}^{\mathbf{c}}$ in the sense of $\delta^{\mathbf{p}}$ are deduced from evaluated manipulation task parameters $\boldsymbol{\chi}_{k}^{\mathrm{e}}$. To do so, given a set of manipulation control parameters $\chi_{k}^{\mathbf{e}}$, horizon $\chi_{k}^{\mathbf{c}}$ are minimizing $\sum_{i=1}^{N} \omega_{\mathbf{p}} \delta_{k+i}^{\mathbf{p}}+\omega_{\mathbf{p}}^{h}\left\|\mathbf{u}_{i}\right\|^{2}$, and hence 
writes with Eq. (3)

$$
\chi_{k}^{\mathbf{c}}=-\left(\mathbf{P}_{\mathbf{u}}^{\mathbf{d i s} T} \mathbf{P}_{\mathbf{u}}^{\mathrm{dis}}+\frac{\omega_{\mathbf{p}}^{h}}{\omega_{\mathbf{p}}} I_{2 N \times 2 N}\right)^{-1} \mathbf{P}_{\mathbf{u}}^{\mathrm{dis}}{ }^{T}\left[\mathbf{P}_{\mathbf{x}}^{\mathrm{dis}} \hat{\mathbf{c}}_{k}-\left(\overline{\mathcal{P}}_{k}-\mathbf{D}_{k}\right)\right]
$$

where $\overline{\mathcal{P}}_{k} \equiv\left[\begin{array}{lll}\overline{\mathbf{p}}_{k+1} & \cdots & \overline{\mathbf{p}}_{k+N}\end{array}\right]^{T}$ denotes the horizon of ZMP references. Results in the following section where obtained with a Nelder-Mead simplex algorithm $^{11}$ run over $\chi^{\mathbf{e}}$ minimizing $f$ as defined in Eq. (13).

\section{Results}

The validity of this control method is assessed through the simulation of a humanoid robot performing two concurrent tracking tasks: it maintains biped balance while following a pre-defined path with its right hand ( $c f$ left hand side of Fig. 2) where is applied a known external effort in lateral and longitudinal directions as shown in Fig. 3.

Simulated experiments are performed using Arboris-Python, ${ }^{12}$ an opensource dynamic simulator developed at ISIR with the Python programming language, involving a rather accurate model of the iCub robot ${ }^{13}$ with 38 degrees of freedom and 4 contact points at each foot. Four controllers are compared:

(1) $C 1$ : ZMP Preview Control ${ }^{7}$ with $\omega_{\mathbf{p}}=1 . e^{6}$ and $\omega_{\mathbf{p}}^{h}=1$,

(2) $C$ 2: ZMP Preview Control accounting for external force at the hand $\mathbf{F}$ as directly applied on the CoM (ie $\mathbf{F}^{e, c}=\mathbf{F}$ ) with Eq. (14),

(3) C3: Optimized Control Parameters minimizing Eq. (13) with $\omega_{\mathbf{e}}=$ $1 . e^{7}, \omega_{\mathbf{p}}=1 . e^{6}, \omega_{\mathbf{e}}^{h}=1 . e 1$ and $\omega_{\mathbf{p}}^{h}=1$,

(4) $C 4$ : Identical to $C 3$ with $\omega_{\mathbf{e}}=1 . e^{6}, \omega_{\mathbf{p}}=1 . e^{7}, \omega_{\mathbf{e}}^{h}=1 . e 1$ and $\omega_{\mathbf{p}}^{h}=1$.

Controller $C 3$ prioritizes the manipulation tracking performance over the ZMP task, and $C 4$ is parameterized symmetrically. Cumulated normalized tracking errors for both tasks and the four controllers are presented on the right hand side of Fig. 2.

First, these results show the noticeable gain in balance provided by the introduction of an approximation (considered to be applied on the CoM) of the external effort. Second, the preponderance of the manipulation task in the controller $C 3$ leads to a significant increase in the tracking performance of the hand though causing a slight prejudice to balance, while symmetric parameters lead to weaker gains but for both tracking tasks.

An intuitive trend in the stiffness strategy is computed online by controllers $C 3$ and $C 4$ : as shown in Fig. 4 the stiffness parameter $K$ tends to 

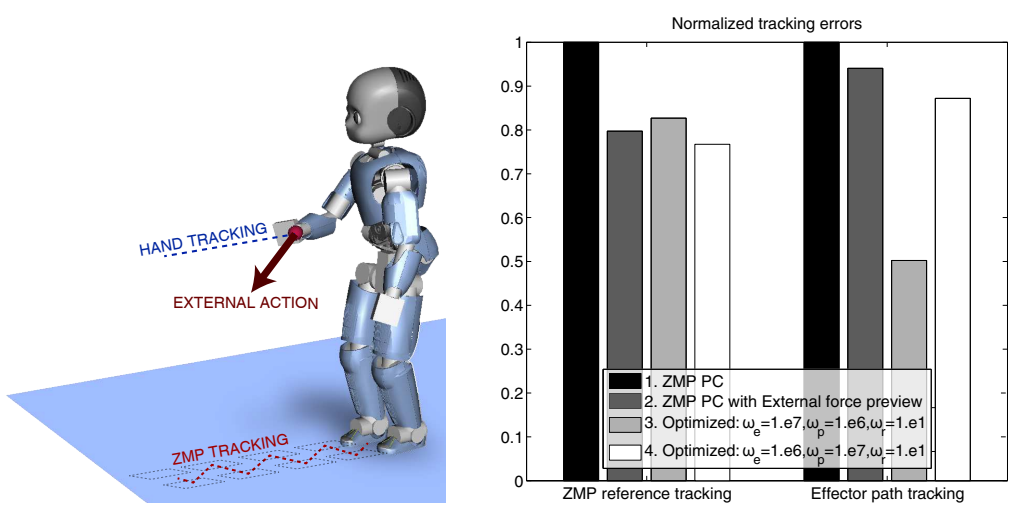

Fig. 2. Two disturbed tracking tasks: manipulation while walking and their normalized cumulated error for four controllers

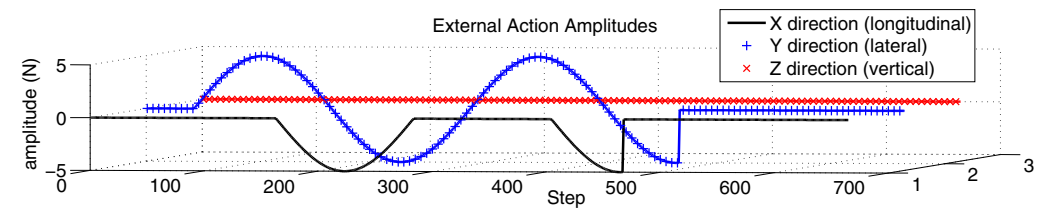

Fig. 3. External action applied on the hand in logitudinal and lateral directions

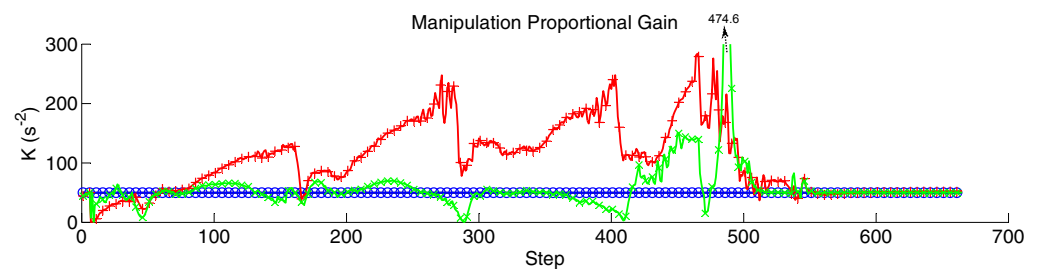

Fig. 4. Control strategy for the manipulation activity - solid: $C 1$, circle: $C 2$, plus: $C 3$ and cross: $C 4$

be set higher than the reference $\bar{K}=50 s^{-2}$ by the controller $C 3$ whereas $C 4$ tends to loosen the manipulation task stiffness under $\bar{K}$ in favor of balance.

A detailed view of the ZMP lateral position is shown in Fig. 5. The decrease in ZMP tracking error resulting from the consideration of the external action is noticeable (eg at steps 250 and 450), and allows for an overall gain in balance. However, balance failure-prone short-term effects as visible at 


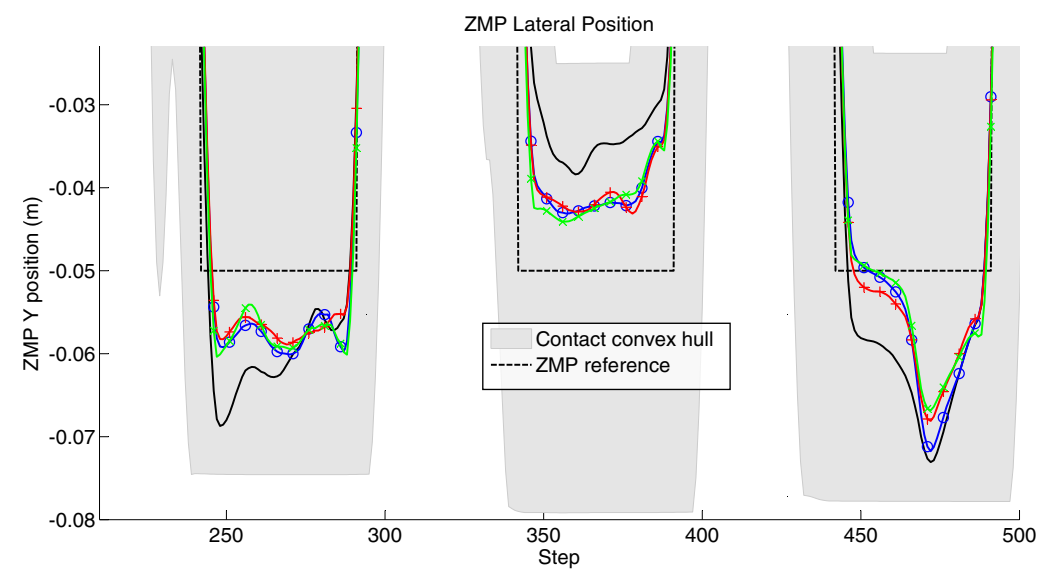

Fig. 5. Detailed evolution of the Zero-Moment Point in the lateral direction - solid: $C 1$, circle: $C 2$, plus: $C 3$ and cross: $C 4$

step 475 require a better estimation and adaptation of the transmission of the external action on the CoM that controllers $C 3$ and $C 4$ provide.

\section{Conclusion}

This work highlights the benefits of considering the coupling between concurrent tasks such as manipulation and balance. Focus has been put on identifying at a high level the influence of manipulation control parameters over the transmission of external actions towards the center of mass of a humanoid robot. This identification allows for an online adaptation of both simultaneous tasks in order to improve their performance based on a preview of external disturbances and their effects on selected criteria.

The resulting formulation produces a robust and parameterizable controller identifying in a predictive manner an optimal control strategy in favor of antagonist objectives.

We propose as future works a deeper study of the interdependence between locomotion and manipulation in a preview control framework.

\section{Acknowledgment}

This work was partially supported by the French Ministry of Higher Education and Research, by the European funds for regional development (FEDER3) for the ANIPEV project and by the RTE company through its chair "Robotics Systems for field intervention in constrained environments" hold by Vincent Padois. 


\section{References}

1. J. Paillard, L'intégration sensori-motrice et idéomotrice, in Traité de Psychologie Expérimentale, eds. M. Richelle, J. Requin and M. Robert (Presses Universitaires de France, 1994) pp. 925-961.

2. A. Wing, J. R. Flanagan and J. Richardson, Experimental Brain Research 116, 122 (1997).

3. N. Hogan, Impedance control: An approach to manipulation, in American Control Conference, 1984.

4. L. Love and W. Book, Environment estimation for enhanced impedance control, in Proceedings of the IEEE International Conference on Robotics \& Automation, 1995.

5. A. Albu-Schaffer, C. Ott, U. Frese and G. Hirzinger, Cartesian impedance control of redundant robots: Recent results with the dlr-light-weight-arms, in Proceedings of the IEEE International Conference on Robotics 83 Automation, 2003.

6. A. N. Venkat, Distributed model predictive control : theory and applications, $\mathrm{PhD}$ thesis2006.

7. S. Kajita, F. Kanehiro, K. Kaneko, K. Kajiwara, K. Harada, K. Yokoi and H. Hirukawa, Biped walking pattern generation by using preview control of zero-moment point, in Proceedings of the IEEE International Conference on Robotics \& Automation, 2003.

8. P.-B. Wieber, Trajectory free linear model predictive control for stable walking in the presence of strong perturbations, in IEEE-RAS International Conference on Humanoid Robots, 2006.

9. O. Khatib, A unified approach to motion and force control of robot manipulators: The operational space formulation, in IEEE Journal on Robotics 86 Automation, 1987.

10. J. Salini, V. Padois and P. Bidaud, Synthesis of complex humanoid wholebody behavior: a focus on sequencing and tasks transitions, in Proceedings of the IEEE International Conference on Robotics $\&$ Automation, 2011.

11. J. Nelder and R. Mead, The Computer Journal 7, 308 (1965).

12. S. Barthelemy, J. Salini and A. Micaelli, Arboris-python.

13. G. Sandini, G. Metta and D. Vernon, The icub cognitive humanoid robot: An open-system research platform for enactive cognition, in 50 Years of Artificial Intelligence, Lecture Notes in Computer Science (Springer, 2007) pp. 358369 . 